\title{
Dynamic changes of the extracellular matrix after acute tako-tsubo cardiomyopathy
}

\author{
Trevor Ahearn ${ }^{1 *}$, Baljit Jagpal ${ }^{2}$, Donnie Cameron², Bernice $\mathrm{K} \mathrm{Ng}^{3}$, Caroline Scally ${ }^{3}$, David M Higgins ${ }^{4}$, \\ John Horowitz ${ }^{5}$, Michael P Frenneaux ${ }^{3}$, Dana K Dawson ${ }^{3}$ \\ From 18th Annual SCMR Scientific Sessions \\ Nice, France. 4-7 February 2015
}

\section{Background}

We have recently demonstrated that cardiac energetic impairment and global myocardial edema persists for at least 4 months after an acute episode of Tako-tsubo cardiomyopathy (TTC). The aim of the current study was to evaluate the regional edema acutely and the status of the extracellular matrix at follow up

\section{Methods}

Eleven patients (10F, mean age $56 \pm 16 \mathrm{yrs}$ ) with a clear diagnosis of ST-elevation TTC and emotional trigger were prospectively enrolled and underwent cardiac magnetic resonance acutely (day $0-3$ ) and after 4 months on a Philips 3T Achieva scanner. Native 3-3-5 (MOLLI) T1 mapping was applied acutely, and both native and postcontrast T1 mapping were performed at 4 months followup. Eleven healthy controls underwent only native T1 mapping. T1 maps were: generated using in-house software - written in IDL (Exelis. Boulder CO, USA); quality controlled with chi-square maps; and imported into Segment (Medviso, Lund University, Sweden), where T1 values were generated for 16 segments. Extracellular volumes (ECV) were calculated for the follow-up scan using:

$\mathrm{ECV}=(1$-hermatocrit $)\left(\Delta \mathrm{R}_{1 \text { myocardium }} / \Delta \mathrm{R}_{1 \text { blood }}\right)$

Segments were grouped according to their wall motion (WM) on the acute scan (normal/abnormal).

\section{Results}

From the acute to the follow-up scan, the LVEF improved from $54 \pm 12 \%$ to $66 \pm 6 \%$, whereas LV mass index decreased from $77 \pm 15 \mathrm{~g} / \mathrm{m}^{2}$ to $68 \pm 14 \mathrm{~g} / \mathrm{m}^{2}$, both $\mathrm{p}<0.05$.
At the acute scan, native T1 of abnormal WM segments was significantly longer compared with $\mathrm{T} 1$ from normal WM segments $(1270 \pm 95$ vs $1225 \pm 43 \mathrm{~ms}$, $\mathrm{p}<0.05)$ and both were significantly increased compared to healthy controls $(1188 \pm 16, \mathrm{p}<0.05)$.

At the follow-up scan, ECV was increased to a similar extent both in segments that were dysfunctional and those that were normally contracting in the acute phase ( $33 \%$ and $34 \%$ respectively, $\mathrm{p}=0.05$ ).

\section{Conclusions}

We demonstrate oedema in both normal and abnormally contracting segments in patients with acute TTC and a similar degree of extracellular expansion at follow-up.

\section{Funding}

Tenovus Scotland. Grant number G13/10.

\section{Authors' details}

${ }^{1}$ Medical Physics, NHS Grampian, Aberdeen, UK. ${ }^{2}$ ABIC, Aberdeen University, Aberdeen, UK. ${ }^{3}$ Cardiology, Aberdeen University, Aberdeen, UK. ${ }^{4}$ Philips Healthcare, Guildford, UK. ${ }^{5}$ Cardiology, Queen Elizabeth Hospital, Adelaide, SA, Australia.

Published: 3 February 2015

doi:10.1186/1532-429X-17-S1-P259

Cite this article as: Ahearn et al: Dynamic changes of the extracellular matrix after acute tako-tsubo cardiomyopathy. Journal of Cardiovascular Magnetic Resonance 2015 17(Suppl 1):P259.

${ }^{1}$ Medical Physics, NHS Grampian, Aberdeen, UK

Full list of author information is available at the end of the article 